\title{
Efeito de diferentes concentrações salinas na redução do tempo de cocção de feijão (Phaseolus vulgaris L.)
}

\author{
Juliano Garcia Bertoldo \\ Jefferson Luís Meirelles Coimbra* \\ Cristian Berto da Silveira \\ Diego Toaldo \\ Universidade do Estado de Santa Catarina (UDESC), Centro de Ciências Agroveterinárias (CAV), \\ Instituto de Melhoramento e Genética Molecular (IMEGEM) \\ Av. Camões 2090, CEP 88520-000, Lages - SC, Brasil \\ *Autor para correspondência \\ coimbrajefferson@cav.udesc.br
}

Submetido em 05/09/2007

Aceito para publicação em 07/05/2008

\section{Resumo}

O objetivo deste trabalho foi verificar o efeito de diferentes concentrações salinas, no intuito de avaliar o tempo de cocção em grãos de feijão envelhecidos. O experimento foi conduzido nas dependências do Instituto de Melhoramento e Genética Molecular (IMEGEM/UDESC), sob delineamento de blocos ao acaso, com duas repetições num esquema fatorial 2 x 4 (duas cultivares e quatro doses de $\mathrm{NaCl}$ ). As cultivares utilizadas foram uma do grupo carioca (Pérola) e outra do grupo preto (Uirapuru). Os grãos, após a pesagem, foram hidratados nas diferentes concentrações de cloreto de sódio $(\mathrm{NaCl})$, sem adição de $\mathrm{NaCl}(0 \mathrm{~g})$ e com adição de $\mathrm{NaCl}(5 \mathrm{~g}, 10 \mathrm{~g}$ e $25 \mathrm{~g}$ ) durante 12 horas a $25^{\circ} \mathrm{C}$. Após o embebimento, os grãos foram submetidos ao teste de cocção, utilizando o aparelho cozedor de Mattson. Foi possível determinar as concentrações de $\mathrm{NaCl}$ nas quais ocorreram maior redução dos tempos de cocção. Para o cultivar Pérola o tempo de cocção foi reduzido em 31 minutos, pela adição de $11 \mathrm{~g}$ de $\mathrm{NaCl}$, enquanto o cultivar Uirapuru teve seu tempo de cocção reduzido em 25 minutos na adição de $16,75 \mathrm{~g}$ de $\mathrm{NaCl}$.

Unitermos: Phaseolus vulgaris L., tempo de cocção, $\mathrm{NaCl}$

\section{Abstract}

Effect of different salt concentrations on the reduction of bean (Phaseolus vulgaris) cooking time. The purpose of this work was to examine the effect of different saline concentrations on the cooking time of aged bean seeds. This experiment was conducted at the Molecular Genetics and Improvement Institute (IMEGEM / CAV), employing random block design with two repetitions, in a 2 x 4 factorial arrangement (two cultivars and four doses of $\mathrm{NaCl}$ ). Two cultivars were tested, one belonging to the carioca group (Pérola) and the other belonging to the black group (Uirapuru). After weighing, the grains were imbibed in different sodium chloride $(\mathrm{NaCl})$ concentrations $(0 \mathrm{~g}, 5 \mathrm{~g}, 10 \mathrm{~g}$ and $25 \mathrm{~g})$ for 12 hours at $25^{\circ} \mathrm{C}$. Subsequently, these grains were submitted to a cooking test, using the Mattson cooking apparatus. It was then possible to determine the $\mathrm{NaCl}$ concentrations in which the cooking time presented a significant reduction. The Pérola cultivar cooking time was reduced by 31 minutes by the addition of $11 \mathrm{~g}$ of $\mathrm{NaCl}$ and the Uirapuru cooking time was reduced by 25 minutes by the addition of $16.75 \mathrm{~g}$ of $\mathrm{NaCl}$.

Key words: Phaseolus vulgaris L., cooking time, $\mathrm{NaCl}$ 


\section{Introdução}

A cultura de feijão ocupa uma posição de destaque no cenário mundial. É a espécie mais cultivada no mundo entre as do gênero Phaseolus, tendo o Brasil como o maior produtor e ao mesmo tempo o maior consumidor, onde de acordo com Pereira (1999), é consumido em praticamente todos os estados do país, sendo cultivado durante todos os meses do ano em quase todo o território nacional.

Com as mudanças no cotidiano, o tempo de preparo do feijão deve ser reduzido, no sentido de atender parte da população que não encontra disponibilidade de tempo para um cozimento prolongado. Nesse caso, cultivares com menores tempos de cozimento proporcionam economia de tempo e de energia (Yokoyama e Stone, 2000). Além disso, períodos prolongados de cozimento causam mudanças estruturais em nível celular, provocando perda de nutrientes (Wassimi et al., 1988).

A qualidade das sementes de feijão, que chegam até o consumidor, depende de algumas características como, a época de colheita, manejo da época de colheita, condições de armazenamento e tecnologia de processamento (Kigel, 1999). Muitas vezes até chegar ao consumidor, tais fatores podem afetar as características culinárias, resultando num maior tempo de cozimento dos grãos. Nesse caso, a busca por procedimentos que possam viabilizar a diminuição no tempo de cocção em feijão é fundamental para a melhor aceitação do produto final pelos consumidores. De acordo com Costa et al. (2001), o tempo de cozimento é fator fundamental para a aceitação de um cultivar de feijão pelos consumidores, pois a disponibilidade para o preparo das refeições é, muitas vezes, restrita. Ainda, o tempo prolongado de cozimento do legume não estimula o consumidor, devido à conveniência, o custo e ao valor nutritivo (Urga et al., 2006).

Existe uma correlação negativa entre a absorção de água pelos grãos e o tempo de cocção, ou seja, quanto maior for a absorção de água, menor será o tempo de cozimento do grão. Segundo Kigel (1999), grãos de feijão com maior tempo de cozimento, tendem a absorver menos água do que aqueles que cozinham mais rapidamente. Ainda segundo o mesmo autor, o grau de associação entre absorção de água e tempo de cozimento é relativamente baixo $(\mathrm{r}=0,37)$. Apesar da baixa correlação, a hidratação de grãos de feijão pode representar uma diminuição significativa no tempo de cozimento. A capacidade de absorção da água pelos grãos, antes do cozimento, tem sido utilizada, visto que a capacidade de cocção está relacionada à rápida absorção (Garcia-Vela e Stanley, 1989). Por outro lado, a utilização do teste da capacidade de absorção da água pelos grãos, como indicativo do tempo de cozimento, tem sido questionada devido à baixa correlação encontrada (Carbonell et al., 2003).

A possibilidade de redução no tempo de cocção de grãos quando hidratados, viabilizou o planejamento de metodologias alternativas com o mesmo propósito. Dentre essas metodologias, a hidratação de grãos de feijão em soluções salinas é discutida em alguns trabalhos (Paredes-López et al., 1990; Diniz et al., 2001; Khetarpaul et al., 2005) de forma a viabilizar a cultura do feijão, objetivando grãos com menores tempos de cocção. A adição de elementos químicos na água durante a cocção ou na hidratação tem sido recomendada para reduzir o tempo de cocção (Khetarpaul et al., 2005).

Nesse sentido, este trabalho buscou verificar o feito da hidratação de grãos de feijão em diferentes concentrações salinas, no intuito de reduzir o tempo de cocção.

\section{Material e Métodos}

O experimento foi conduzido nas dependências do Instituto de Melhoramento e Genética Molecular (IMEGEM/CAV), sendo utilizadas sementes de feijão obtidas da safra 2006/07 na área experimental do mesmo local. O delineamento foi o de blocos ao acaso, com duas repetições num esquema fatorial $2 \times 4$ (dois cultivares e quatro doses de $\mathrm{NaCl}$ ).

Os cultivares utilizados são registrados no Serviço Nacional de Proteção de Cultivares (SNPC), sendo uma do grupo carioca (Pérola) e outra do grupo preto (Uirapuru). As doses de cloreto de sódio $(\mathrm{NaCl})$ foram: sem adição de $\mathrm{NaCl}(0 \mathrm{~g})$, com adição de $5 \mathrm{~g}, 10 \mathrm{~g}$ e $25 \mathrm{~g}$. A relação gramas/volume foi de $1 / 4$, sendo utilizadas 
no experimento $50 \mathrm{~g}$ de grãos para $200 \mathrm{ml}$ de água destilada.

Os grãos após a pesagem foram hidratados nas diferentes concentrações durante 12 horas a $25^{\circ} \mathrm{C}$, seguindo a metodologia proposta por Paredes-Lopez et al. (1991). Após o embebimento, os grãos foram submetidos ao teste de cocção, utilizando o aparelho cozedor de Mattson, adaptado por Proctor e Watts (1987). O aparelho é constituído por 25 estiletes verticais com peso de $90 \mathrm{~g}$, terminando com uma ponta de $1 \mathrm{~mm}$ de diâmetro. Os estiletes ficam apoiados sob os grãos de feijão. $\mathrm{O}$ aparelho, com os 25 grãos, foi colocado dentro de uma panela com água destilada fervente (temperatura padronizada de $95^{\circ} \mathrm{C}$ ) e na medida em que ocorre o cozimento, os estiletes perfuram os grãos. O tempo de cocção é estabelecido quando 13 estiletes dos 25 perfuram os grãos $(50 \%+1)$.

O modelo estatístico utilizado neste experimento foi:

$$
y_{i j k}=m+b_{k}+c_{i}+s_{j}+c s_{i j}+e_{i j k}
$$

Fatores experimentais:

i) Cultivar (C); ii) Solução salina (S)

Fator de unidade: i) Bloco (B)

Efeitos expressos na equação do modelo estatístico: $\mathrm{m}$ : média; $\mathrm{b}_{\mathrm{k}}$ : efeito do bloco; $\mathrm{c}_{\mathrm{i}}$ : efeito do cultivar; $\mathrm{s}_{\mathrm{j}}$ : efeito da solução salina; $\mathrm{cs}_{\mathrm{ij}}$; efeito da interação $\mathrm{CxS}$; $\mathrm{e}_{\mathrm{ijk}}$ : erro.

Os dados foram submetidos à análise de variância pelo teste $F$ ao nível de $5 \%$ de probabilidade de erro. Os graus de liberdade foram desdobrados, através do efeito simples, e posteriormente foram ajustadas regressões para as interações, através do programa estatístico SAS 9.1.3 (2007).

\section{Resultados e Discussão}

Os resultados da análise de variância (Tabela 1) evidenciaram efeito significativo da interação entre os fatores cultivar versus solução salina.
TABELA 1: Análise de variância para o tempo de cozimento de sementes feijão. Lages, 2007.

\begin{tabular}{lcc}
\multicolumn{1}{c}{$\begin{array}{c}\text { Fontes de } \\
\text { variação }\end{array}$} & $\begin{array}{c}\text { Graus de } \\
\text { liberdade }\end{array}$ & $\begin{array}{c}\text { Quadrado } \\
\text { médio }\end{array}$ \\
Cultivar (C) & 1 & 5,06 \\
Solução salina (S) & 3 & $131,56^{*}$ \\
CxS & 3 & $74,89^{*}$ \\
Erro & 5 & 9,18 \\
\hline Total & 15 & - \\
C.V.\% & - & 8,55 \\
\hline
\end{tabular}

*Significativo em nível a 5\% de probabilidade de erro pelo teste $\mathrm{F}$.

Nesse caso, os graus foram desdobrados, através do efeito simples fixando um fator e variando o outro (Tabela 2). De acordo com Hinkelmann e Kempthorne (1994), o procedimento de variar um fator por vez, via de regra, se aplica quando o objetivo é estabelecer uma lei fundamental, o que conduziria ao conhecimento detalhado do efeito de um fator, quando os outros são mantidos constantes. O desdobramento dos graus de liberdade possibilitou verificar o efeito de um fator dentro do nível do outro, e nesse caso, todos foram significativos (Tabela 2).

TABELA 2: Desdobramento dos graus de liberdade para a interação cultivar versus doses de sal $(\mathrm{CxS})$ para os cultivares Pérola e Uirapuru em diferentes doses de cloreto de sódio $(\mathrm{NaCl})$.

\begin{tabular}{lcc}
\hline \multicolumn{1}{c}{ Genótipo } & $\begin{array}{c}\text { Graus de } \\
\text { Liberdade }\end{array}$ & $\begin{array}{c}\text { Quadrado } \\
\text { médio }\end{array}$ \\
\hline Pérola & 3 & $103,00^{*}$ \\
Uirapuru & 3 & $133,45^{*}$ \\
Erro & 5 & 9,18 \\
\hline
\end{tabular}

Significativo em nível a $5 \%$ de probabilidade de erro pelo teste $\mathrm{F}$.

Sendo a interação significativa, e um dos fatores de natureza quantitativa (concentrações salinas), foi determinado o grau do polinômio através de contrastes, e de acordo com a significância, foi ajustada uma equação de regressão linear ou quadrática para os fatores que foram significativos pelo efeito simples (Tabela 3 ). Nos 
casos em que os tratamentos forem de níveis de um fator quantitativo, o apropriado é decompor os graus de liberdade em componentes polinomiais ortogonais (Chew, 1976).

TABELA 3: Contrastes para determinação do grau do polinômio para os cultivares Pérola e Uirapuru em diferentes doses de cloreto de sódio $(\mathrm{NaCl})$.

\begin{tabular}{lcc}
\hline Contrastes & $\begin{array}{c}\text { Graus de } \\
\text { liberdade }\end{array}$ & $\begin{array}{c}\text { Quadrado } \\
\text { médio }\end{array}$ \\
\hline Cultivar Pérola - linear & 1 & $56,25^{*}$ \\
$\begin{array}{l}\text { Cultivar Pérola - quadrá- } \\
\text { tico }\end{array}$ & 1 & $36,75^{*}$ \\
Cultivar Uirapuru - linear & 1 & $289,00^{*}$ \\
$\begin{array}{l}\text { Cultivar Uirapuru - qua- } \\
\text { drático }\end{array}$ & 1 & $93,33^{*}$ \\
Erro & 5 & 9,18 \\
\hline
\end{tabular}

* Significativo em nível a $5 \%$ de probabilidade de erro pelo teste $\mathrm{F}$.

Os resultados revelam a influência da hidratação com solução salina para o tempo de cocção sendo que, a adição de sal durante a hidratação dos grãos de feijão, possibilitou uma redução no tempo de cocção nos grãos dos cultivares estudados até certo ponto (Figura 1).

De acordo com a figura 1, para o cultivar Pérola, o ponto de mínimo para a dose de $\mathrm{NaCl}$ foi de $11 \mathrm{~g}$. $\mathrm{O}$ tempo inicial de cocção foi de 37,81 min, reduzindo para cerca de $31 \mathrm{~min}$ na concentração de $11 \mathrm{~g}$ e aumentando posteriormente ao se elevar a concentração de $\mathrm{NaCl}$.

O comportamento de o cultivar Uirapuru foi similar ao do cultivar Pérola, porém o ponto de mínimo foi com a concentração de $16,75 \mathrm{~g}$, reduzindo de $45,95 \mathrm{~min}$ sem adição de $\mathrm{NaCl}$, para cerca de $25 \mathrm{~min}$ com adição de $\mathrm{NaCl}$. Tal fato demonstra que há uma redução significativa no tempo de cocção dos grãos de feijão estudados, quando hidratados em solução salina. Entretanto, a hidratação com maior concentração salina, propiciou um aumento no tempo necessário para cocção dos grãos. Sendo assim, doses de $11 \mathrm{~g}$ de $\mathrm{NaCl}$ para o cultivar Pérola e doses de $16,75 \mathrm{~g}$ de $\mathrm{NaCl}$ para o cultivar Uirapuru são suficientes para a redução no tempo de cocção. Ainda, o cultivar Pérola revelou um aumento no tempo de cocção na concentração de $25 \mathrm{~g}$ quando comparado com a dose zero (sem adição de sal), porém para o cultivar Uirapuru, o tempo de cocção sem adição de sal foi maior do que a dose $25 \mathrm{~g}$ de $\mathrm{NaCl}$. Tal fato pode estar corroborando com o efeito significativo da interação entre genótipos e doses de $\mathrm{NaCl}$.

Alguns trabalhos buscam esclarecer a eficácia da hidratação com soluções salinas no intuito de reduzir o tempo de cozimento em grãos. Diniz et al. (2001) estudando a caracterização química e tecnológica de
Pérola

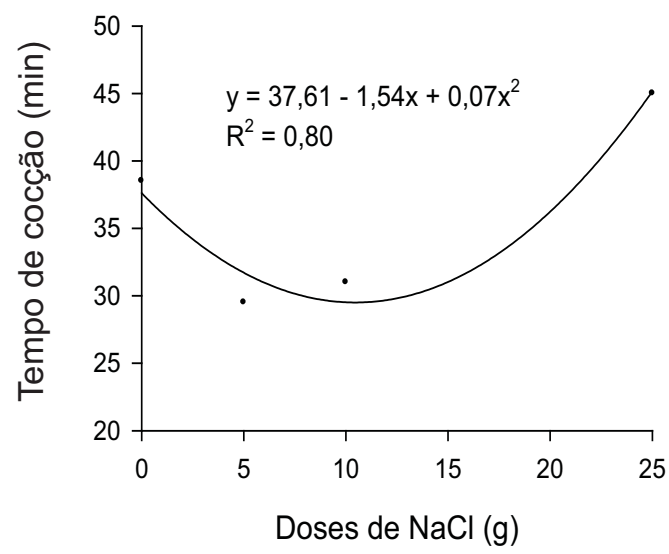

Uirapuru

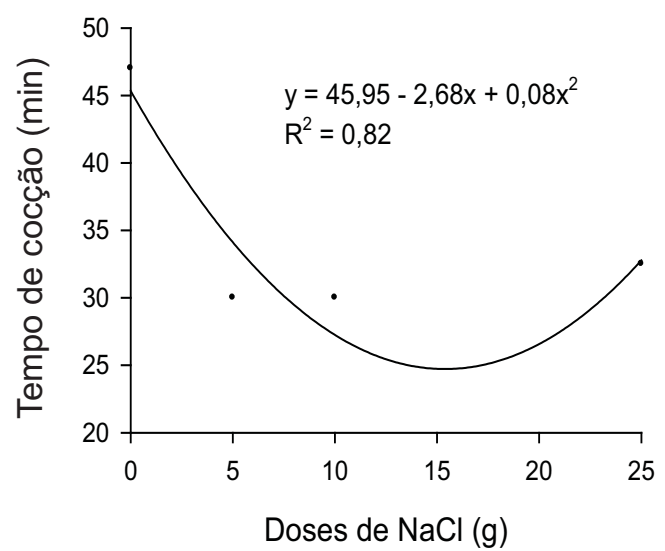

FIGURA 1: Comportamento dos cultivares Pérola e Uirapuru em diferentes doses de cloreto de sódio ( $\mathrm{NaCl})$ para o tempo de cocção. 
4 variedades de feijão, verificou diferenças entre a cocção entre as variedades submetidas à cocção sem sal (cloreto de sódio) e com a adição de sal (cloreto de sódio). Porém, os resultados expostos no trabalho citado não possibilitam visualizar uma ação definida do sal quanto ao tempo de cocção dos grãos, pois, embora tenha ocorrido redução destes em três dentre as quatro variedades estudadas, em uma delas, a Manteiguinha, ocorreu um aumento do tempo de cocção dos grãos. Em contrapartida, Paredes-López et al. (1990) verificou uma redução no tempo de cocção com a adição de $\mathrm{NaCl}$ combinado com $\mathrm{NaHCO}_{3}$. Khetarpaul et al. (2005), estudando o efeito do pré-tratamento em soja com solução salina no tempo de cocção, concluiu que a hidratação em soluções salinas, como por exemplo, carbonato e bicarbonato de cálcio, reduz o tempo de cocção significativamente.

Amudança decorrente no tempo de cocção a partir da adição de sal na hidratação ocorre devido a modificações na estrutura dos cotilédones. Conforme verificado por ParedesLopéz et al. (1991), mudanças que ocorrem na lamela média são responsáveis pela redução no tempo de cocção, ocorrendo uma separação nas células dos cotilédones, fato que contribui para a redução do tempo de cocção. Tal ruptura pode facilitar a cocção, no sentido de favorecer uma rápida entrada de água nos grãos hidratados em solução salina e posteriormente aquecidos em água fervente. Segundo Urga et al. (2006), o tempo de cozimento em legumes é afetado pela permeabilidade do tegumento e dos cotilédones das sementes em água fervente. Durante a cocção dos grãos de feijão, a protopectina nativa existente na lamela média forma uma pectina solúvel que despolimeriza rapidamente durante o aquecimento e facilita a entrada rápida de água e o seu movimento para as células do cotilédone (Stanley e Aguilera, 1985). Segundo Khetarpaul et al. (2005), diferenças entre tratamentos com adição de sal na solução salina ou não, podem ser verificadas pelo rompimento de componentes intercelulares, bem como a absorção de água pelas sementes aumentar por causa da contribuição de sólidos para o potencial osmótico e potenciais mátricos das soluções.

Com relação à textura dos grãos, na medida em que se aumentam as concentrações de $\mathrm{NaCl}$, ocorrem mudanças na morfologia dos grãos. Os tratamentos sem adição salina e na concentração de $5 \mathrm{~g}$ de $\mathrm{NaCl}$ não apresentaram grãos desidratados. Porém, nos tratamentos com as concentrações 10 e $25 \mathrm{~g}$ de $\mathrm{NaCl}$, os grãos apresentaram um aspecto rugoso. Ainda, nas duas últimas concentrações, a textura dos grãos estava mais macia. Entre as culturas de grãos, o feijoeiro é a que exibe o mais alto nível de variabilidade quanto à cor, tamanho e forma da semente, sendo que estas características influenciam as pessoas quanto à preferência por determinada variedade (Carneiro et al., 2005). Os mesmos autores, em seus estudos concluíram que, para os cultivares estudados, existem diferenças significativas em relação aos atributos sensoriais de seus grãos, tais como sabor característico, gosto amargo, dureza, granulosidade. De acordo com Teixeira et al. (2001), atualmente, para o feijão, as características de grãos que mais merecem atenção são o tamanho, o formato e, sobretudo a coloração do tegumento.

Para a aceitação de cultivares pelos consumidores, é necessário que esta tenha um tempo de cozimento reduzido, e, além disso, que reúna alguns aspectos em níveis de aceitabilidade, tais como sabor, aroma e textura. A aceitação de sementes de feijão pelo consumidor depende do tamanho, cor, forma e qualidades culinárias, como o tempo de cozimento, presença de grãos inteiros, textura, sabor, aroma e total de sólidos após o cozimento (Dalla Corte et al., 2003). Singh e Rao (1995) verificaram em seus trabalhos que, de modo geral, a hidratação de sementes em solução salina, promove uma boa aceitabilidade mediante o tempo de cocção, sabor, aroma e textura dos grãos. Para tanto, realizaram análises sensoriais e classificaram os tratamentos de acordo com as propriedades, numa escala de 1 a 4 (1-ruim; 2-média; 3-bom; 4-excelente). Da mesma forma, Khetarpaul et al. (2005), em soja, agrupou as propriedades culinárias numa escala sensorial, indicando que alguns tratamentos com solução salina apresentaram uma boa aceitação dentre as propriedades culinárias estudadas.

Isto indica que a hidratação de grãos em soluções salinas pode ser uma alternativa em busca de redução do caráter tempo de cocção, e conseqüentemente, na melhoria nas qualidades culinárias de grãos armazenados durante 120 dias ou mais, uma vez que, em cultivares comerciais, é muito variável. Avaliando linhagens de 
feijão de ensaio nacional para o tempo de cocção, do grupo preto e carioca, Carneiro et al. (1999) encontraram variabilidade entre os genótipos para todos os caracteres avaliados, dentre estes o escurecimento do grão.

Mediante tais fatos, existe a necessidade em se avaliar as qualidades culinárias dos grãos de feijão, tanto para o caráter tempo de cocção, quanto para as qualidades sensoriais, como textura, cor, sabor e aroma. Segundo Carneiro et al. (2005), nos últimos anos os pesquisadores do Programa de Melhoramento Genético do Feijoeiro têm reconhecido a importância das características físicas e sensoriais dos grãos de cultivares de feijão na sua aceitação pelos consumidores. Ainda, para os mesmos autores, fica evidente a necessidade da caracterização sensorial dos grãos de cultivares de feijão que já são recomendados para o cultivo e daqueles que estão para serem recomendados.

Os resultados permitem concluir que as concentrações salinas reduziram o tempo de cocção dos grãos de feijão. A hidratação dos grãos de feijão durante 12 horas em solução salina reduziu significativamente o tempo de cocção. Avaliações sensoriais para outras qualidades culinárias, como sabor, textura e aroma, são necessárias, antes de indicar um melhor tratamento com solução salina.

\section{Referências}

Carbonell, S. A. M.; Carvalho, L.; Pereira, V. R. 2003. Qualidade tecnológica de grãos de genótipos de feijoeiro cultivados em diferentes ambientes. Bragantia, 62 (3): 369-379.

Carneiro, J. D. S; Araújo, G. A. A.; Carneiro, J. E. S.; Del Peloso, M. J.; Carneiro, G. E. S.; Carneiro, P. C. S. 1999. Potencial tecnológico dos grãos de linhagens de feijão (Phaseolus vulgaris L.). Resumos expandidos da $6^{\text {a }}$ RENAFE - Reunião Nacional de Pesquisa de Feijão, v.1, Salvador, Brasil, p.408-411.

Carneiro, J. C. S; Minim, V. P. R.; Souza, M. M.; Carneiro, J. E. S.; Araújo, G. A. A. 2005. Perfil sensorial e aceitabilidade de cultivares de feijão (Phaseolus vulgaris L.). Ciência e Tecnologia de Alimentos, 25 (1): 18-24.

Chew, V. 1976. Comparing treatment means: a compendium. Hortscience, 11 (4): 348-357.

Costa, G. R.; Ramalho, M. A. P.; Abreu, A. F. B. 2001. Variabilidade para absorção de água nos grãos de feijão do germoplasma da UFLA. Ciência e Agrotecnologia, 25 (4): 1017-1021.

Dalla Corte, A.; Moda-Cirino, V.; Scholz, M. B. S.; Destro, D. 2003. Environment effect on grain quality in early common bean cultivars and lines. Crop Breeding and Applied Biotechnology, 3 (3): 193-202.

Diniz, M. C.; Silva, C. L.; Aragão, N. L. L.; Muniz, M. B.; Ferreira, G. M.; Oliveira, R. T. 2001. Caracterização química e tecnológica de 4 variedades de feijão macasar verde (Vigna unguiculata (L.) walp) comercializadas e consumidas no município de Campina Grande - PB. Revista Brasileira de Produtos Agroindustriais, 3 (1): 91-100.

Garcia-Vela, L. A.; Stanley, D. W. 1989. Water-holding capacity in hard-to-cook bean (P. vulgaris L.): effect of $\mathrm{pH}$ and ionic strength. Journal of Food Science, 54 (4): 1080-1081.

Hinkelmann, K.; Kempthorne, O. 1994. Design and analysis of experiments: Introduction to experimental design. John Wiley \& Sons, New York, USA, 495pp.

Khetarpaul, N.; Goyal, R.; Garg, R. 2005. Effect of salt solution pretreatment on the cooking quality and consumer acceptability of soy dhal. British Food Journal, 107 (5): 344-352.

Kigel, J. 1999. Culinary and nutritional quality of Phaseolus vulgaris seeds as affected by evironmental factors. Biotechnologie Agronomie Society Enviroment, 3 (4): 205-209.

Paredes-Lopez, O.; Carabez-Tryo, A.; Palmer-Tirodo, L.; RyesMoreno, C. 1991. Influence of hardening procedure and soaking solution on cooking quality of common beans. Plant Foods for Human Nutrition, 41 (1): 155-164.

Pereira, P. A. A. 1999. A cultura do feijão no Brasil: situação atual e perspectivas. In: Fancelli, A. L. \& Dourado Neto, D. (coords). Feijão irrigado: Estratégias básicas de manejo. Escola Superior de Agricultura "Luiz de Queiroz", Piracicaba, Brasil, p.1-8.

Proctor, J. R.; Watts, B. M. 1997. Development of a modified Mattson bean cooker procedure based on sensory panel cookability evaluation. Canadian Institute of Food Science and Technology, 20 (1): 9-14.

SAS. 2007. SAS 9.1.3 (TS1M3) for Windows Microsoft. SAS Institute Inc., Cary, USA, sem paginação.

Singh, U.; Rao, P. V. 1995. Quick-cooking dhal of pigeonpea as influenced by salt solution and enzyme pretreatments. Journal of Food Science and Technology, 32 (2): 122-125.

Stanley, D. W.; Aguilera, J. M. 1985. A review of textural defects in cooked reconstituted legumes - the influence of structure and composition. Journal of Food Biochemistry, 9: 277-323.

Teixeira, F. F.; Baldoni, A. B.; Santos, J. B. 2001. Controle genético de características associadas à coloração do grão do feijão no cruzamento Rosinha x Esal 693. Anais do $1^{\circ}$ Congresso Brasileiro de Melhoramento de Plantas, Goiânia, Brasil, CD-ROM.

Urga, K.; Fufa, H.; Biratu, E.; Gebretsadik, M. 2006. Effects of blanching and soaking on some physical characteristics of grass pea (Lathyrus sativus). African Journal of Food Agriculture Nutrition and Development, 6 (1): 1-17.

Yokoyama, L. P.; Stone, L. F. 2000. Cultura do feijoeiro no Brasil: Características da produção. Embrapa Arroz e Feijão, Santo Antônio de Goiás, Brasil, 75pp.

Wassimi, N. N.; Hosfield, G. L.; Uebersax, M. A. 1988. Combining ability of tannin content and protein characteristics of raw and cooked dry beans. Crop Science, 28 (3): 452-458. 